\title{
MS36-02 | Manipulation of the Crystalline and Amorphous Physical States of Pharmaceutical Materials: Possibilities, Limits and Challenges
}

ROCA PAIXAO, Luisa (University of Lille, Villeneuve-d'Ascq, FRA); Correia, Natalia (University of LILLE, VILLENEUVE D'ASCQ, FRA); Ngono Mebenga, Frederic (University of LILLE, VILLENEUVE D'ASCQ, FRA); Willart, Jean-Francois (University of Lille/CNRS, VILLENEUVE D'ASCQ, FRA); Cuello, Gabriel (Institut Laue-Langevin, Grenoble, FRA); Jimenez, Monica (Institut Laue-Langevin, Grenoble, FRA); Affouard, Frederic (University of LILLE, VILLENEUVE D'ASCQ, FRA)

The vast majority of drugs are generally prepared in the solid state (powder, tablets, and capsules) which can exist in multiple physical forms having fundamental different physical properties. Until now, pharmaceutical materials have been developed especially in the crystalline state for obvious reasons of stability. However, this state may often exhibit inadequate solubility or dissolution rate resulting in poor bioavailability, particularly for waterinsoluble compounds. Different solutions have been proposed to overcome this major issue either based on chemical modifications of the drug molecules (salt formation) or physical manipulations of their physical states. For example, an interesting alternative is offered by the amorphous state which exhibits much greater dissolution rate. However, it is intrinsically unstable and capable of recrystallization which obviously negates its advantages. A "stabilization" is thus required and, for a few years, there has been a considerable interest on the so-called amorphous solid dispersions for which the strategy is to disperse pharmaceuticals in the amorphous state into a polymer matrix that hinders its recrystallization.

In this presentation, a review of the different possibilities, limits and challenges of the approaches based on the manipulation of the physical states and aiming to improve properties of pharmaceutical materials in terms of solubility, dissolution and stability will be presented.

This project has received funding from the Interreg 2 Seas programme 2014-2020 co-funded by the European Regional Development Fund under subsidy contract 2S01-059_IMODE. 